\title{
Evolution of Floquet Topological Quantum States in Driven Semiconductors
}

\author{
Andreas Lubatsch ${ }^{1}$ and Regine Frank ${ }^{2,3, a}$ \\ 1 Georg-Simon-Ohm University of Applied Sciences, Keßlerplatz 12, 90489 Nürnberg, Germany \\ 2 Bell Laboratories, 600 Mountain Avenue, Murray Hill, NJ 07974-0636, USA \\ 3 Serin Physics Laboratory, Department of Physics and Astronomy, Rutgers University, 136 Frelinghuysen Road, Piscataway, \\ NJ 08854-8019, USA
}

Received: February 16, 2019 / Revised version: July 21, 2019

\begin{abstract}
Spatially uniform excitations can induce Floquet topological bandstructures within insulators which have equal characteristics to those of topological insulators. Going beyond we demonstrate in this article the evolution of Floquet topological quantum states for electromagnetically driven semiconductor bulk matter. We show the direct physical impact of the mathematical precision of the Floquet-Keldysh theory when we solve the driven system of a generalized Hubbard model with our framework of dynamical mean field theory (DMFT) in the non-equilibrium. We explain the physical consequences of the topological non-equilibrium effects in our results for correlated sysems with impact on optoelectronic applications.
\end{abstract}

PACS. 71.10.-w Theories and models of many-electron systems - 42.50.Hz Strong-field excitation of optical transitions in quantum systems; multi-photon processes; dynamic Stark shift $-74.40+$ Fluctuations 03.75.Lm Tunneling, Josephson effect, Bose-Einstein condensates in periodic potentials, solitons, vortices, and topological excitations - 72.20.Ht High-field and nonlinear effects - 89.75.-k Complex systems

\section{Introduction}

Topological phases of matter [1,2,3] have captured our imagination over the past decades, revealing properties such as robust edge modes and exotic non-Abelian excitations [4,5. Potential applications of periodically driven quantum systems [6] are conceivable from semiconductor spintronics [7] to topological quantum computation [8, topological lasers 9, 10, and random lasers [11 in optics. The discovery of topological insulators in solid-state devices such as $\mathrm{HgTe} / \mathrm{CdTe}$ quantum wells [12,13, and topological Dirac insulators such as $\mathrm{Bi}_{2} \mathrm{Te}_{3}$ and $\mathrm{Bi}_{2} \mathrm{Sn}_{3}$, 14, 15 [16] were milestones in the research of the unique properties of topological phases in technological applications.

Recently, it could be shown that the application of timeperiodic perturbations can induce topological properties in conventional insulators 17, 18 which are trivial in equilibrium. So called Floquet topological insulators include a wide range of physical solid state and atomic realizations, driven both at resonance and off-resonance. These systems display metallic conduction enabled by quasi-stationary states at the edges 19, 20, 17, Dirac cones in three dimensional systems [21,22,23, and Floquet Majorana fermions [24. Novel materials like Graphene are investigated as Floquet fractional Chern insulators $25,26,27$.

Send offprint requests to: regine.frank@googlemail.com, regine.frank@rutgers.edu
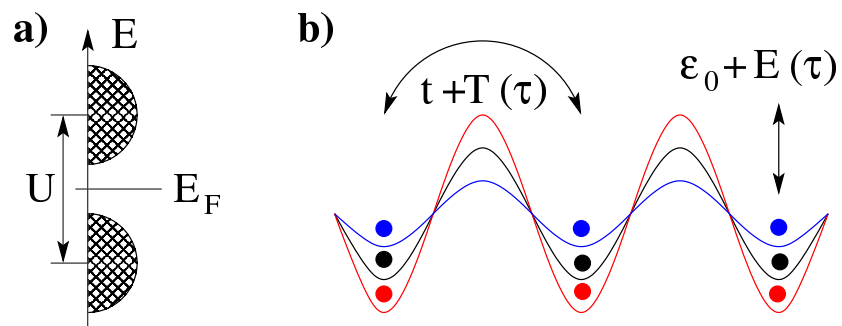

Fig. 1. Insulator to metal transition due to photo-excitation. (a) Schematic split in energy bands by the local Coulomb interaction $U$. The width of the gap is determined symmetrically to the Fermi edge $E_{F}$. (b) A periodic in time driving yields the additional hopping contribution $T(\tau)$ of electrons in the lattice (black) and the local renormalization of the lattice energy, the local potential, $E(\tau)$, as a quasi-energy. The colors in the lattice potential represent the external driving in time.

In this article we show that Floquet topological quantum states evolve in correlated electronic systems of driven conventional semi-conductors at low temperature. The nonequilibrium is in this sense defined by the intense external driving field which induces topologically dressed electronic states as well as dynamical gaps. We show that the mathematical method of the expansion into Floquet modes 28 is leading to results of direct physical impact, when we implement it for modeling the coupling of a classical elec- 


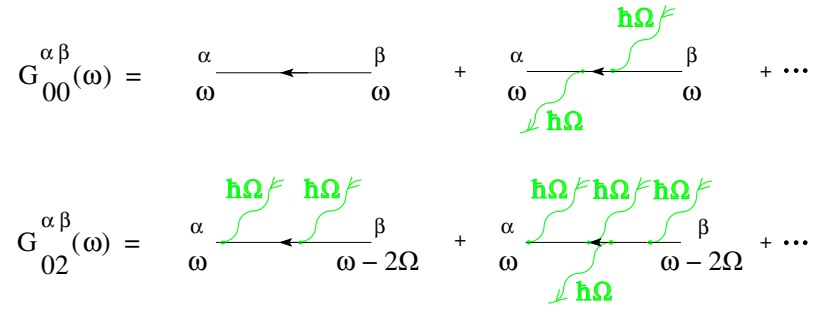

Fig. 2. The contributions to the Floquet Greens function in terms of absorption and emission of external energy quanta $\hbar \Omega_{L}$ are schematically explained. $G_{00}^{\alpha \beta}(\omega)$ represents the sum of all balanced processes, $G_{02}^{\alpha \beta}(\omega)$ describes a net absorption of two photons. $\alpha, \beta$ are Keldysh indices.

tromagnetic external driving field to the correlated quantum many body system. Our results derived by Dynamical Mean Field Theory (DMFT) in the non-equilibrium provide novel insights in topologically induced phase transitions of driven and otherwise conventional 3-dimensional semiconductor bulk matter and insulators.

\section{Quantum Many Body Theory for Floquet Topological States in the Non-Equilibrium}

We consider in this work the wide gap semiconductor bulk as it is driven by a strong periodic in time external field in the optical regime which yields higher-order photon absorption processes. The electronic dynamics of the photoexcitation processes, compare with Fig. 1, is modeled as a generalized driven Hubbard Hamiltonian Eq. (1). The system is solved by applying a Keldysh formalism including the electron-photon interaction in the sense of coupling the electromagnetic field to the electronic dipole and thus to the electronic hopping as an additional kinetic contribution, compare figure 2. We implement a dynamical mean field theory (DMFT), compare with Fig. 3, with a generalized iterative perturbation theory solver (IPT), see Fig. 4. in order to solve the system numerically which will be explained in detail. We start with the introduction of the full interacting Hamiltonian, Eq. (1),

$$
\begin{aligned}
H= & \sum_{i, \sigma} \varepsilon_{i} c_{i, \sigma}^{\dagger} c_{i, \sigma}+\frac{U}{2} \sum_{i, \sigma} c_{i, \sigma}^{\dagger} c_{i, \sigma} c_{i,-\sigma}^{\dagger} c_{i,-\sigma} \\
& -t \sum_{\langle i j\rangle, \sigma} c_{i, \sigma}^{\dagger} c_{j, \sigma} \\
& +i \boldsymbol{d} \cdot \boldsymbol{E}_{0} \cos \left(\Omega_{L} \tau\right) \sum_{<i j>, \sigma}\left(c_{i, \sigma}^{\dagger} c_{j, \sigma}-c_{j, \sigma}^{\dagger} c_{i, \sigma}\right) .
\end{aligned}
$$

In Eq. (1) $c^{\dagger},(c)$ denote the creator (annihilator) of an electron. The subscripts $i, j$ indicate the site, $\langle i, j\rangle$ implies the sum over nearest neighboring sites. The term $\frac{U}{2} \sum_{i, \sigma} c_{i, \sigma}^{\dagger} c_{i, \sigma} c_{i,-\sigma}^{\dagger} c_{i,-\sigma}$ is devoted to repulsion due to the onsite Coulomb interaction $U$ between electrons with opposite spins. The third term $-t \sum_{\langle i j\rangle, \sigma} c_{i, \sigma}^{\dagger} c_{j, \sigma}$ describes
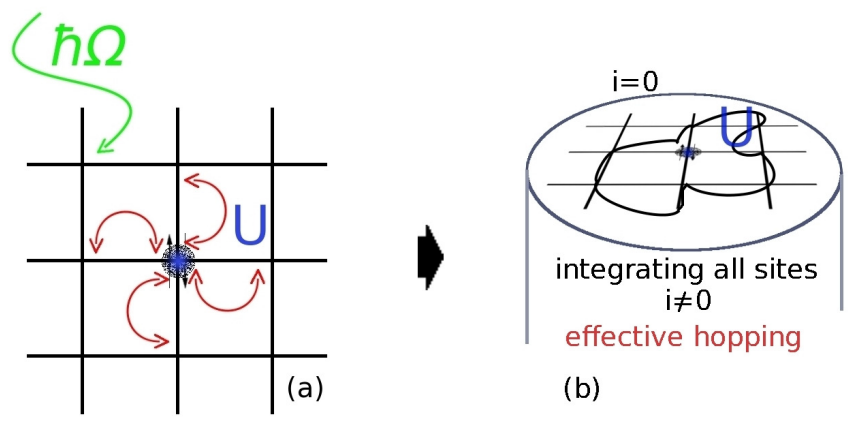

(b)

Fig. 3. Schematic representation of the non-equilibrium dynamical mean field theory. (a) The semiconductor behaves in the considered regime as an insulator: In the non-equilibrium DMFT-scheme optical excitations by external photons of energy $\hbar \Omega$ yield additional electronic hopping processes. (b) DMFT idea: Integration over all lattice sites leads to an effective theory including non-equilibrium excitation. The bath consists of all single sites and the approach is thus self-consistent. The driven electronic system may in principal couple to a surface-resonance or an edge state. The coupling to these states can be enhanced by the external excitation of the electronic many body system.

a standard hopping of electrons with the amplitude $t$ between nearest neighbor sites. Those contributions form the standard Hubbard model which we generalize for our purposes in what follows. The first term $\sum_{i, \sigma} \varepsilon_{i} c_{i, \sigma}^{\dagger} c_{i, \sigma}$ generalizes the Hubbard model with respect to the onsite energy. The electronic on-site energy is noted as $\varepsilon_{i}$. The central feature, the external time-dependent electromagnetic driving is described in terms of the field $\boldsymbol{E}_{0}$ with laser frequency $\Omega_{L}, \tau$. We model the photonic interaction as a coupling of the amplitude of the impinging electromagnetic wave and the electronic dipole operator $\hat{d}$ with strength $|\mathbf{d}|$. The expression $i \boldsymbol{d} \cdot \boldsymbol{E}_{0} \cos \left(\Omega_{L} \tau\right) \sum_{<i j>, \sigma}\left(c_{i, \sigma}^{\dagger} c_{j, \sigma}-c_{j, \sigma}^{\dagger} c_{i, \sigma}\right)$ describes the renormalization of the standard electronic hopping processes, as a possible contribution $T(\tau)$ in Fig. (1), due to interaction with the external pump field.

\subsection{COUPLING Of A CLASSICAL DRIVING FIELD TO A QUANTUM DYNAMICAL SYSTEM}

In order to solve the generalized Hubbard Hamiltonian Eq. (1) for the driven system, we briefly introduce the explicit time dependency of the external field. It yields Green's functions which depend on two separate time arguments.

The double Fourier transform from time to frequency coordinates leads to two frequencies which are chosen as the relative and the center-of-mass frequency [29,30] and we introduce an expansion into Floquet modes

$$
\begin{aligned}
G_{m n}^{\alpha \beta}(\omega) & =\int \mathrm{d} \tau_{1}^{\alpha} \mathrm{d} \tau_{2}^{\beta} e^{-i \Omega_{L}\left(m \tau_{1}^{\alpha}-n \tau_{2}^{\beta}\right)} e^{i \omega\left(\tau_{1}^{\alpha}-\tau_{2}^{\beta}\right)} G\left(\tau_{1}^{\alpha}, \tau_{2}^{\beta}\right) \\
& \equiv G^{\alpha \beta}\left(\omega-m \Omega_{L}, \omega-n \Omega_{L}\right)
\end{aligned}
$$


Generally Floquet 28 states are analogues to Bloch states. Whereas the Bloch state results from a spatially periodic potential the Floquet state is a result of the temporal periodicity $31,32,33,34,35,36,37,29,30,38$. Either induces a specific topological band-structure. The Floquet expansion is introduced in Fig. 2 as an intelligible graphical explanation of what is described in Eq. (2). $(m, n)$ label the Floquet modes, whereas $(\alpha, \beta)$ refer to the branch of the Keldysh contour $( \pm)$ where the respective time argument resides. The result of this process, the Floquet modes or Floquet quasi energies are derived by a Fourier transformation of the periodic in time potential as the principal structure of bands in frequency space. It is basically the analogue procedure in time to the calculation of the band structure due to periodic in space potentials in solid state materials. The physical consequence of the process however is noteworthy. It can be understood as the quantized absorption and emission of energy $\hbar \Omega_{L}$ out of and into the classical external driving field by the driven quantum many body system.

In the analytical case of uncorrelated electrons, $U=0$, the solution for the Green's function $G_{m n}(k, \omega)$ can be found directly by solving the Hamiltonian which eventually reads for the retarded component

$$
G_{m n}^{R}(k, \omega)=\sum_{\rho} \frac{J_{\rho-m}\left(A_{0} \tilde{\epsilon}_{k}\right) J_{\rho-n}\left(A_{0} \tilde{\epsilon}_{k}\right)}{\omega-\rho \Omega_{L}-\epsilon_{k}+i 0^{+}} .
$$

Here $\tilde{\epsilon}_{k}$ represents the dispersion relation which is induced by the external driving field and which is to be distinguished from the lattice dispersion $\epsilon_{k} . J_{n}$ are the cylindrical Bessel functions of integer order, $A_{0}=\boldsymbol{d} \cdot \boldsymbol{E}_{0}$ and $\Omega_{L}$ is the laser frequency as introduced before. The retarded Green's function for the optically excited band electron is then given by

$$
G_{\mathrm{Lb}}^{R}(k, \omega)=\sum_{m, n} G_{m n}^{R}(k, \omega) .
$$

\subsection{NUMERICAL SOLUTIONS: DYNAMICAL MEAN FIELD THEORY IN THE NON-EQUILIBRIUM}

The generalized Hubbard model for the correlated system at hand, $U \neq 0$, in the non-equilibrium, Eq. (1), is numerically solved by means of a single-site Dynamical Mean Field Theory (DMFT) [38, 39, 40, 41, 42, 43, 44 45, 46, 47, 48, 49,50,51,52. As explained in section 2.1 the expansion into Floquet modes in combination with the proper Keldysh description implements the external time dependent classical driving field, and couples it to the quantum many body system. The Floquet-Keldysh DMFT 38,49 is solved with a second order iterative perturbation theory (IPT), the contributing diagrams to the local selfenergy $\Sigma^{\alpha \beta}$ are explained in Fig. 4. The Green's function $G_{\mathrm{Lb}}^{R}(k, \omega)$ for the interaction of the band electron with the external laser, Eq. (4), is characterized by the wave vector $k$, which describes the periodicity of the lattice, and it depends on the electronic frequency $\omega$ and the external
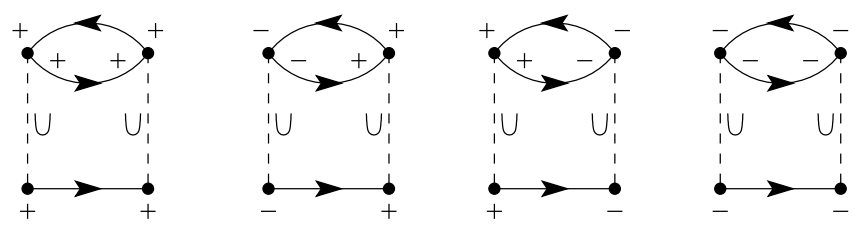

Fig. 4. Local self-energy $\Sigma^{\alpha \beta}$ within the iterated perturbation theory (IPT). The used IPT is a second order diagrammatic solver with respect to the electron electron interaction $U$ and it is here generalized to non-equilibrium, \pm signs are indicating the respective branch of the Keldysh contour. The solid lines represent the bath-Green's function, the so called Weiss-field $\mathcal{G}^{\alpha \beta}$, see ref. 49].

driving frequency $\Omega_{L}$, see Eq. 2, that are captured in the Floquet indices $(m, n)$. For the requirements of our system the DMFT self-consistency relation, the actual centerpiece of the DMFT scheme, assumes the form of a matrix equation of the non-equilibrium Green's functions which is of dimension $2 \times 2$ in regular Keldysh space and of dimension $n \times n$ in Floquet space. The resulting numerical algorithm proves to be efficient and stable also for all values of the Coulomb interaction $U$.

Whereas in previous work $38,49,48$ we considered an additional kinetic energy contribution due to a lattice vibration, here we take into account a coupling of the microscopic electronic dipole moment to an external electromagnetic field 29 30 for the correlated system. This requires the introduction of the quantum-mechanical expression for the electronic dipole operator $\hat{d}$, see the last term r.h.s. Eq. 1, 1 , $\boldsymbol{d} \cdot \boldsymbol{E}_{0} \cos \left(\Omega_{L} \tau\right) \sum_{<i j>, \sigma}\left(c_{i, \sigma}^{\dagger} c_{j, \sigma}-c_{j, \sigma}^{\dagger} c_{i, \sigma}\right)$. This contribution is conceptionally different from the generic kinetic hopping of the third term of Eq.(1). The coupling $\hat{d} \cdot \boldsymbol{E}_{0} \cos \left(\Omega_{L} \tau\right)$ generates the factor $\Omega_{L}$ that cancels the $1 / \Omega_{L}$ in the renormalized cylindrical Bessel function in Eq. (7) of ref. 49] under the assumption of the Coulomb gauge, $\boldsymbol{E}(\tau)=-\frac{\partial}{\partial \tau} \boldsymbol{A}(\tau)$, which reads in Fourier space as $\boldsymbol{E}\left(\Omega_{L}\right)=i \Omega_{L} \cdot \boldsymbol{A}\left(\Omega_{L}\right)$. The consistency check of the Floquet sum is discussed in section 3.3 here in detail.

It has been shown by [41] that the coupling of an electromagnetic field modulation to the onsite electronic density $n_{i}=c_{i, \sigma}^{\dagger} c_{i, \sigma}$ alone as an effect in the unlimited three dimensional translationally invariant system, can be gauged away. The effect for this type of coupling can be absorbed in an overall shift of the local potential while no additional dispersion is reflecting any induced functional dynamics of the system. Thus such a system 25. 55. is obviously not going to show any topological effects in the sense of a topological insulator or a Chern insulator. By contrast, the coupling of the external electromagnetic field modulation to the dipole moment of the elementary charge, and thus to the hopping, see Eq. (1), in the sense of a kinetic energy of the fermions, cannot be gauged away and it is causing the evolution of topological states in the three dimensional unlimited system. Line three of Eq. (1) formally represents the electromag- 

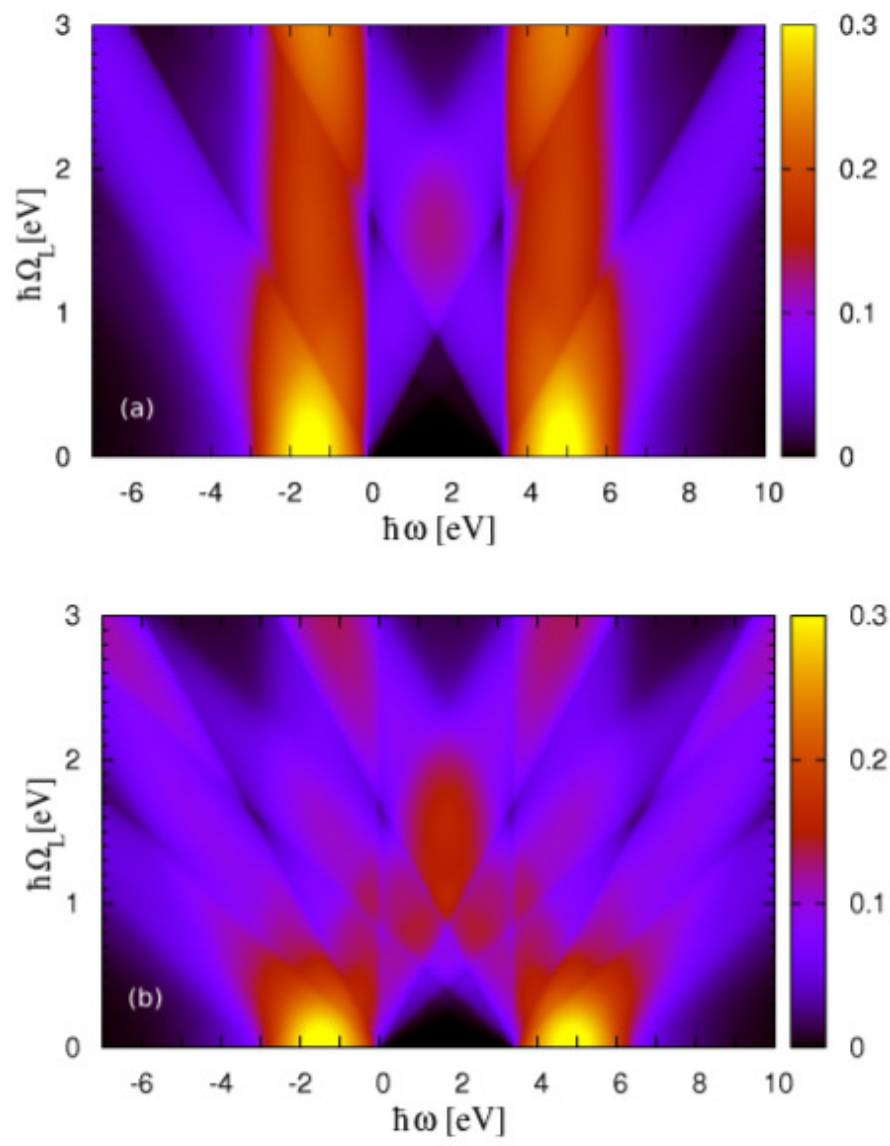

Fig. 5. Energy spectra of Floquet topological quantum states of the semiconductor bulk in the non-equilibrium. (a) The evolution of the LDOS in the non-equilibrium for varying excitation laser frequencies $\Omega_{L}$ is displayed. The excitation intensity of $5.0 \mathrm{MW} / \mathrm{cm}^{2}$ is kept constant. The original bandgap in the equilibrium, see Fig. 1, is vanishing with rising driving frequency and dressed states emerge due to the non-equilibrium AC-Stark effect [53,54]. The split bandstructure is replaced by a Floquet fan which evolves with increasing external excitation frequency. The intersection of bands is observed and the formation of topological subgaps, see e.g. at $\hbar \Omega_{L}=1.75 \mathrm{eV}$ can be observed. (b) The evolution of the LDOS in the nonequilibrium for varying excitation laser frequencies $\Omega_{L}$ is displayed, the excitation intensity of $10.0 \mathrm{MW} / \mathrm{cm}^{2}$ is assumed. Spectral weight is shifted to a multitude of Floquet-bands which clearly emerge, while the original split band characteristics almost vanishes apart from the near-gap band edges. The Floquet bands overlap with each other and a variety of Floquet gaps are formed. Multiple induced band edges are found and their crossing points can be identified, where topologically induced transitions are possible, especially the generation of higher harmonics can be enhanced at such points. For a detailed discussion see section 3

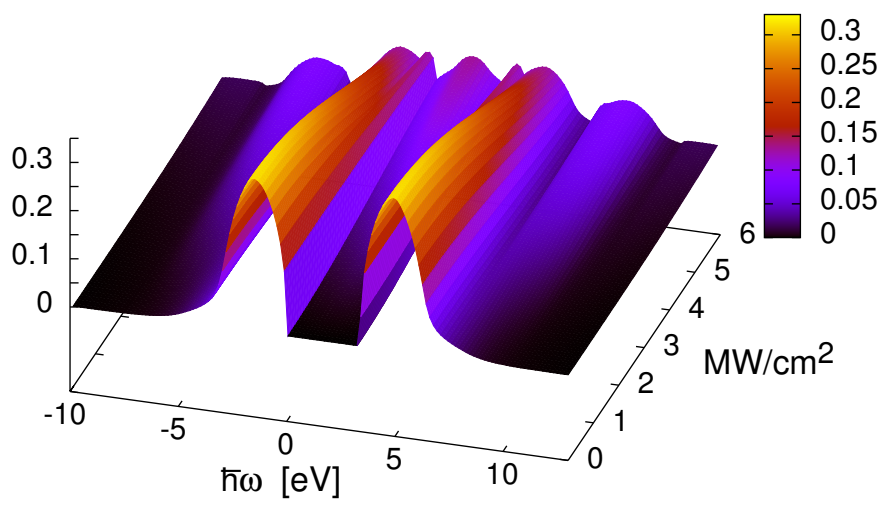

Fig. 6. Energy spectra of Floquet topological quantum states of the semiconductor bulk in the non-equilibrium. The evolution of the LDOS is displayed for the single excitation energy of $\hbar \Omega_{L}=1.75 \mathrm{eV}$, wavelength $\lambda=710.0 \mathrm{~nm}$ and an increasing external driving intensity up to $6.0 \mathrm{MW} / \mathrm{cm}^{2}$. Spectral weight is shifted by excitation to emerging Floquet sidebands and a sophisticated sub gab structure is developed. In the nonequilibrium such topological effects in correlated systems are non-trivial. The bandgap in equilibrium is $3.38 \mathrm{eV}$, the Fermi edge is $1.69 \mathrm{eV}$, the width of each band is $3.0 \mathrm{eV}$. For a detailed discussion see section 3 .

netically induced kinetic contribution

$$
\begin{array}{r}
i \boldsymbol{d} \cdot \boldsymbol{E}_{0} \cos \left(\Omega_{L} \tau\right) \sum_{<i j>, \sigma}\left(c_{i, \sigma}^{\dagger} c_{j, \sigma}-c_{j, \sigma}^{\dagger} c_{i, \sigma}\right)= \\
e \sum_{\boldsymbol{r}} \hat{j}_{i n d}(\boldsymbol{r}) \cdot \boldsymbol{A}(\boldsymbol{r}, \boldsymbol{\tau})
\end{array}
$$

which is equivalent to a kinetic contribution of the photo-induced charge current dependent in space with $\boldsymbol{r}$

$$
\boldsymbol{j}_{i n d}(\boldsymbol{r})_{\delta}=-\frac{t}{i} \sum_{\sigma}\left(c_{\boldsymbol{r}, \sigma}^{\dagger} c_{\boldsymbol{r}+\delta, \sigma}-c_{\boldsymbol{r}+\delta, \sigma}^{\dagger} c_{\boldsymbol{r}, \sigma}\right)
$$

The temporal modulation of the classical external electrical field in (111) direction always comes along with a temporally modulated magnetic field contribution $\boldsymbol{B}(\boldsymbol{r}, \tau)=$ $\nabla \times \boldsymbol{A}(\boldsymbol{r}, \tau)$ with $\boldsymbol{B}\left(\boldsymbol{r}, \Omega_{L}\right)$ in Fourier space, as a simple consequence of Maxwells equations. In what follows we derive the non-equilibrium local density of states (LDOS) as the central result of DMFT which comes along with the dynamically evolving life-time of non-equilibrium states as the inverse of the imaginary part of the self-energy $\tau \sim 1 / \Im \Sigma^{R}$. A time reversal procedure derived by an external field will in any case not be able to revise the non-equilibrium effect. The light-electron coupling will be modified and the overall profoundly differing material characteristics, the conductivity and the polarization of the excited matter in the non-equilibrium are preventing any time-reversal process in the sense of closing the Floquet 
fan again in this regime. The initially impinging electromagnetic field thus causes a break of the time-reversal symmetry, the current ultimately leads to the acquisition of a non-zero Berry flux. A Wannier-Stark type ladder 56 is created that can be characterized by the Berry phase [57] in the sense of a Chern or a winding number and the $\mathbb{Z}_{2}$ invariants in three dimensions respectively 58 .

\section{Floquet Spectra for Driven Semiconductors}

From the numerically computed components of the Green's function, we define the local density of states $N\left(\omega, \Omega_{L}\right)$ where momentum is integrated out and Floquet indices are summed

$$
N\left(\omega, \Omega_{L}\right)=-\frac{1}{\pi} \sum_{m n} \int \mathrm{d}^{3} k \operatorname{Im} G_{m n}^{R}\left(\mathbf{k}, \omega, \Omega_{L}\right) .
$$

In the following we show results for optically excited semiconductor bulk matter, with a band gap in the equilibrium of $3.38 \mathrm{eV}$, typical parameters for $\mathrm{ZnO}$. $\mathrm{ZnO}$ [59 $60,61,62$ is a very promising material for the construction of micro-lasers, quantum wells and optical components as well as for the engineering of ultrafast switches in certain geometries and in connection to other topological insulators. It is broadly investigated in the non-centrosymmetric wurtzite configuration as well as in the centrosymmetric, cubic, rocksalt configuration 63, 64, it's bandgap is estimated to be of $2.4 \mathrm{eV}$ up to $6.1 \mathrm{eV}$ depending on various factors as the pressure during the fabrication process. Either crystal configuration is a good candidate for the production of second or higher order harmonics under intense external excitations 65. Thus it is of high interest for this work.

\subsection{Evolution of Floquet Topological Quantum States in the Non-Equilibrium}

In Fig. 5 we investigate a wide gap semi-conductor structure, the band gap in equilibrium is assumed to be $3.38 \mathrm{eV}$, which shall be exposed to an external periodic-in-time driving field. The system is so far considered as pure bulk, so we are investigating pure Floquet topological effects in the non-equilibrium. The excitation intensity is considered to be $5.0 \mathrm{MW} / \mathrm{cm}^{2}$ in the results of Fig. 5 (a) and $10.0 \mathrm{MW} / \mathrm{cm}^{2}$ in the results of Fig. 5(b). DMFT as a solver for correlated and strongly correlated electronics as such is a spatially independent method and thus it is designed to derive bulk effects, whereas all k-dependencies have been integrated as a fundamental aspect of the methodology. Thus we are not analyzing the k-resolved information of the Brillouin zone, DMFT results in one, two and three dimensions are independent of any spatial information as long as no artificial coarse graining with a novel length scale in the sense of finite elements or finite volumes is included. The sheer fact however, that the energy dependent LDOS profoundly changes with a varying excitation frequency and with a varying excitation intensity as well, proves that also the underlying k-dependent bandstructure is topologically modulated and a non-trivial topological structure of the Hilbert space is generated by external excitation. Our system in equilibrium is fully periodic in space and time. The dependent-in-time external electrical field generates an temporally modulated magnetic field and thus a dynamical Wannier-Stark effect. Floquet states are generated. They are the temporal analogue to Bloch states, and the argumentation by Zak [56] applies for the generation of the Berry phase $\gamma_{m}$ when a solid is exposed to an externally modulated electromagnetic potential [57,66, 67,68]. The Floquet quasi-energies, the topology, see Fig. 2, are labeled by the Floquet modes in dependency to the external excitation frequency, and to the external excitation amplitude. The topological invariants, the Chern number as a sum over all occupied bands $n=\sum_{m=1}^{\nu} n_{m} \neq 0$ and the $\mathbb{Z}_{2}$ invariants include the Berry flux $n_{m}=1 / 2 \pi \int d^{2} \boldsymbol{k}\left(\nabla \times \gamma_{m}\right)$. The winding number is also associated consistently with the argument of collecting a non-zero Berry flux. The driving frequency is considered to be smaller than the width of the semiconductor gap in equilibrium. While the system is excited and thus evolving in non-equilibrium, a Berry phase is acquired and a non-zero Berry flux und thus a non-zero Chern number are characterizing the topological bandstructure as non-trivial. For one dimensional models [56, 69 it is well acknowledged that with the reduction of the external excitation frequency $\Omega_{L}$ novel sets of Floquet bands, so called replica, emerge in the spectrum, which are attributed a quantised change of the Berry phase $\gamma=\pi$. In three dimensions the situation as such is more complicated, the Berry phase is usually attributed to the Wyckoff positions of the crystal and the Brioullin zone respectively [56, and as such it cannot be derived by the pure form of the DMFT. $\boldsymbol{k}$-dependent information can be derived by so called real-space or cluster DMFT solutions (R-DMFT or CDMFT) 70,71,72,73, however up to our best knowledge a powerful solution for true three-dimensional systems in the non-equilibrium is not existing in presence. These thoughts however do not stand against the logical consequence that a system out of equilibrium acquires a non-zero Berry phase. The inclusion of interactions leads to a Mott-type gap which closes due to the superposition of the crossing Floquet bands, however also the opening of non-equilibrium induced Mott-gap replica can be found for $\Omega_{L}=1.69 \mathrm{eV}$, which is half of the width of the Mottgap. The replica are complete at $\Omega_{L}=3.38 \mathrm{eV}$, see Fig. 5 (a). For the increase of $\boldsymbol{E}_{0}$ these gap replica are again crossed by the next order of Floquet sidebands, however their width at $\Omega_{L}=3.38 \mathrm{eV}$ is confirmed. It is easily to understand that the closing of the Mott-gap and the opening of side Mott-gaps, so called replica, in the spectrum due to topological excitation are classified as non-trivial topological effect. In general the classification of correlated topological systems is a very active research field [25,74, 75. We refer to the section 3.3 in this article, where we show that in our theoretical results also at the crossing points the contribution by each Floquet mode is distinguishable and we analyse it as such. For the investigation 
of the local density of states and the occupation number, the filling, as well as the life-times of these states, an artificial cut-off of the Floquet series, as is described in the literature, does not make sense from the numerical physics point of view of the DMFT in frequency space, since it would hurt basically conservation laws. The cut-off would lead to a drift of the overall energy of the system, see section 3.3. We refer thus to the bulk-boundary correspondence [2,3] which predicts that the results of this work for bulk will be observed in a pump-probe experiment at the surface of the semiconductor sample. In what follows we discuss des evolution of Floquet topological states for an increasing external driving frequency $\Omega_{L}$, see Fig.(5), and for an increasing amplitude of the driving, see Fig.(6), of the correlated system, and we discuss the physical consequences and possible applications.

\subsection{Topologically Induced Generation of Higher Harmonics and Optical Transparency}

When we increase the external excitation energy from $0 \mathrm{eV}$ to $3.0 \mathrm{eV}$, we find the evolution of Floquet topological quantum states, as well as the topologically induced Floquet band gaps for bulk matter. In Fig. 5(a) the development of a very clear Floquet fan for the valence as well as for the conduction band of the correlated matter in the non-equilibrium is found. Valence and conduction band split in a multitude of Floquet sub-bands which cross each other. For excitation energies up to $2.5 \mathrm{eV}$ the original band gap is subsequently closing, the first crossing point inside the semiconductor gap along the Fermi edge is found at $0.9 \mathrm{eV}$. With increasing excitation intensity, see Fig. 5(b), higher order Floquet side bands emerge and we find the next prominent crossing point at the Fermi-edge for $0.45 \mathrm{eV}$. Band edges of higher order Floquet bands form crossing points with those of the first order, e.g. for the excitation energy $0.7 \mathrm{eV}$ the crossing points of the first side bands (02) with the higher number side bands are found at the atomic energy of $1.08 \mathrm{eV}$ and $2.3 \mathrm{eV}$ above the valence band edge and thus deep in the gap of the semiconductor. Semiconductors are well known for fundamental absorption at the band edge of the valence band. What we find here is that the absorption coefficient of the semiconductor is topologically modulated and non-trivial transitions at the crossing points of Floquet-valence subbands and Floquet-conduction subbands become possible and significant. Having in mind that a higher order Floquet subband is usually physically reached by absorption or generation of higher harmonic procedures we find that there is a high probability existing for topologically induced direct transitions from the fundamental to higher order bands in those spectral positions where a Floquet band-edge crosses the inner band edge of the equilibrium valence band. At any bandedge in some sense directional scattering can be expected if the life-times of the states are on the scale of the expected scattering procedures. In general the optical refractive index will be topologically modulated, electromagnetically induced transparency may be observable for high ampli- tude excitations. The topologically induced Floquet bands overlap and cross each other. Consequentially very pronounced features such as narrow subgaps are formed in the LDOS. In the excited semiconductor Floquet replica of valence and conduction bands are formed and the dispersion is renormalized. We also find regions, e.g. for excitation energies from $\hbar \Omega_{L}=0.9 \mathrm{eV}$ up to $\hbar \Omega_{L}=1.3 \mathrm{eV}$ and from $\hbar \Omega_{L}=1.8 \mathrm{eV}$ up to $\hbar \Omega_{L}=2.2 \mathrm{eV}$ which show a topologically induced metallic phase. The dressed states are the result of the Franz-Keldysh effect $76,77,78,79,80$ or AC-Stark effect which is well known for high intensity excitation of semiconductor bulk and quantum wells [53. 54 .

From the methodological viewpoint of correlated electronics in the non-equilibrium our results can be interpreted as follows. For finite excitation frequencies an instantaneous transition to the topologically induced Floquet band structure and a renormalized dispersion is derived. In the bulk system clear Floquet bands develop, when the sample is excited by an intense electrical field. This is observable in Fig. 5 .

In Fig. 6 we show the same system as in Fig. 5 for constant driving energy of $1.75 \mathrm{eV}$ and an increasing driving intensity up to $6.0 \mathrm{MW} / \mathrm{cm}^{2}$. We find the evolution of side bands and an overall vanishing semiconductor gap, which marks the transition from semi-conductor to a topologically highly tunable and switchable conductor in the nonequilibrium.

At this point we do not investigate with our model the coupling to a geometrical edge or a resonator mode, which would lead for the optical case to additional contributions in Eq. (1) for the mode itself $\hbar \omega_{o} a^{\dagger} a$ and the coupling of this resonator or edge mode $g \sum_{i, \sigma} c_{i, \sigma}^{\dagger} c_{i, \sigma}\left(a^{\dagger}+a\right)$.

$a^{\dagger}$ and $a$ are the creator and the annihilator of the photon, $g$ is the coupling strength of the photonic mode to the electronic system. From our results however we can conclude already that for semi-conductor cavities and quantum wells as well as for structures which enhance so called edge states that may couple to non-equilibrium topological Floquet bands, these geometrical edge or surface resonances will induce an additional topological effect within the full so far excitonic spectrum. It is an additional effect beyond the bulk boundary correspondence. Dressed states may release energy quanta, e.g. light, or an electronic current into the resonator mode [29]. So we expect from our results that such modes may become a very sensible switch in non-equilibrium.

It is to clarify whether novel topological effects in the non-equilibrium due to the geometry may be of technological use, since they modify the full spectrum. For the investigation of $\mathrm{ZnO}$ as a laser material in this regime the influences of surface resonators will be subject to further investigations. It will be on target to find out all the signatures of a topologically protected edge mode in correlated and strongly correlated systems out of equilibrium in the experiment and to define the role of the topologically induced local density of bulk states e.g. for the occurrence of the electro-optical Kerr effect, the magneto-optical Kerr effect (MOKE) and the surface magneto-optical Kerr ef- 
fect (SMOKE). We believe that in correlated many-body systems out of equilibrium the topological edge mode is found to behave in the sense of the bulk boundary correspondence. Those results are expected to become modified or enhanced by a coupling of bulk states with a geometrical surface mode or a resonance.

\subsection{Floquet Sum and Consistency of the Numerical Framework}

An analysis of the numerical validity in terms of the normalized and frequency integrated density of states

$$
N_{i}\left(\Omega_{L}\right):=\int \mathrm{d} \omega N\left(\omega, \Omega_{L}\right)=1
$$

is generally confirmed for summing over Floquet indices up to the order or 10. We discuss in Fig. 7 on the l.h.s. the Floquet contributions as examples with increasing order in steps of $n=0,2,4,6,8,10$. With an increasing order of the Floquet index the amplitude of the Floquet contribution decreases up to the point where the level of precision of the DMFT self-consistency is reached. This is definitely the case for $n=10$ and thus a physical argument to cut the Floquet expansion off for $n=10$. The displayed Floquet contributions $G_{0 \pm n}$ are of course perfectly mirror symmetric with respect to the Fermi edge, whereas the sum of both contributions is directly symmetric with respect to the Fermi edge. This is generally a proof of the validity of the numerical Fourier transformation and the numerical scheme. The order of magnitude for Floquet contributions of higher than $n=4$ is almost falling consistently with the rising Floquet index. We display here results for an external laser with the wavelength of $\lambda=710.0 \mathrm{~nm}$ and the intensity of $3.8 \mathrm{MW} / \mathrm{cm}^{2}$. The semiconductor gap is assumed to be $3.38 \mathrm{eV}$ which corresponds to $\mathrm{ZnO}$ as a laser active material. From the analysis it can be deduced that we include Floquet contributions up to a precision of $10^{-3}$ with regard to their effective difference from the final result which is displayed on the r.h.s of Fig. 7 as the sum up to the order of n. That corresponds to the overall accuracy of the self-consistent numerical approach. Higher order modes do not contribute to the LDOS within the accuracy of the model under the assumption of the given excitation intensities.

The physical interpretation of Fig. 7 is an interesting point in the discussion of the evolution of the topological quantum states. The order of the Floquet contribution $n$ numbers also the evolving Floquet side bands which emerge in the LDOS, compare Fig. 5(a). Whereas the lowest order Floquet contribution $G_{00}$ is symmetric to the Fermi edge but strictly positive, higher order contributions $G_{0 \pm n}$ are mirror symmetric to each other and in sum they can have negative contributions to the result of the LDOS. Their impact decreases with the order. The Floquet contributions as such consequentially do not have a direct physical interpretation however the sum of all contributions equals the material characteristics of the local density of
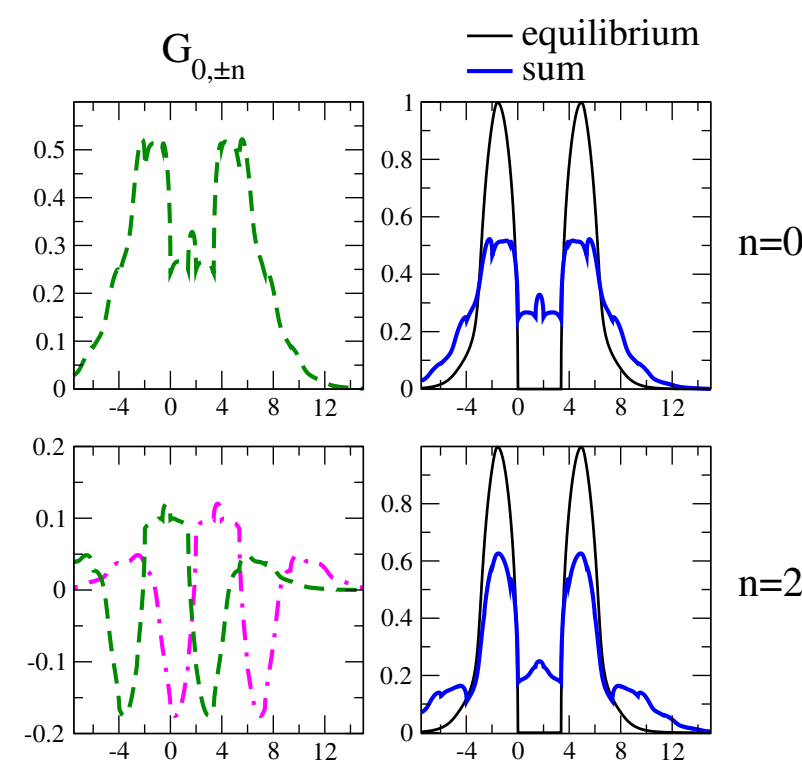

$n=2$
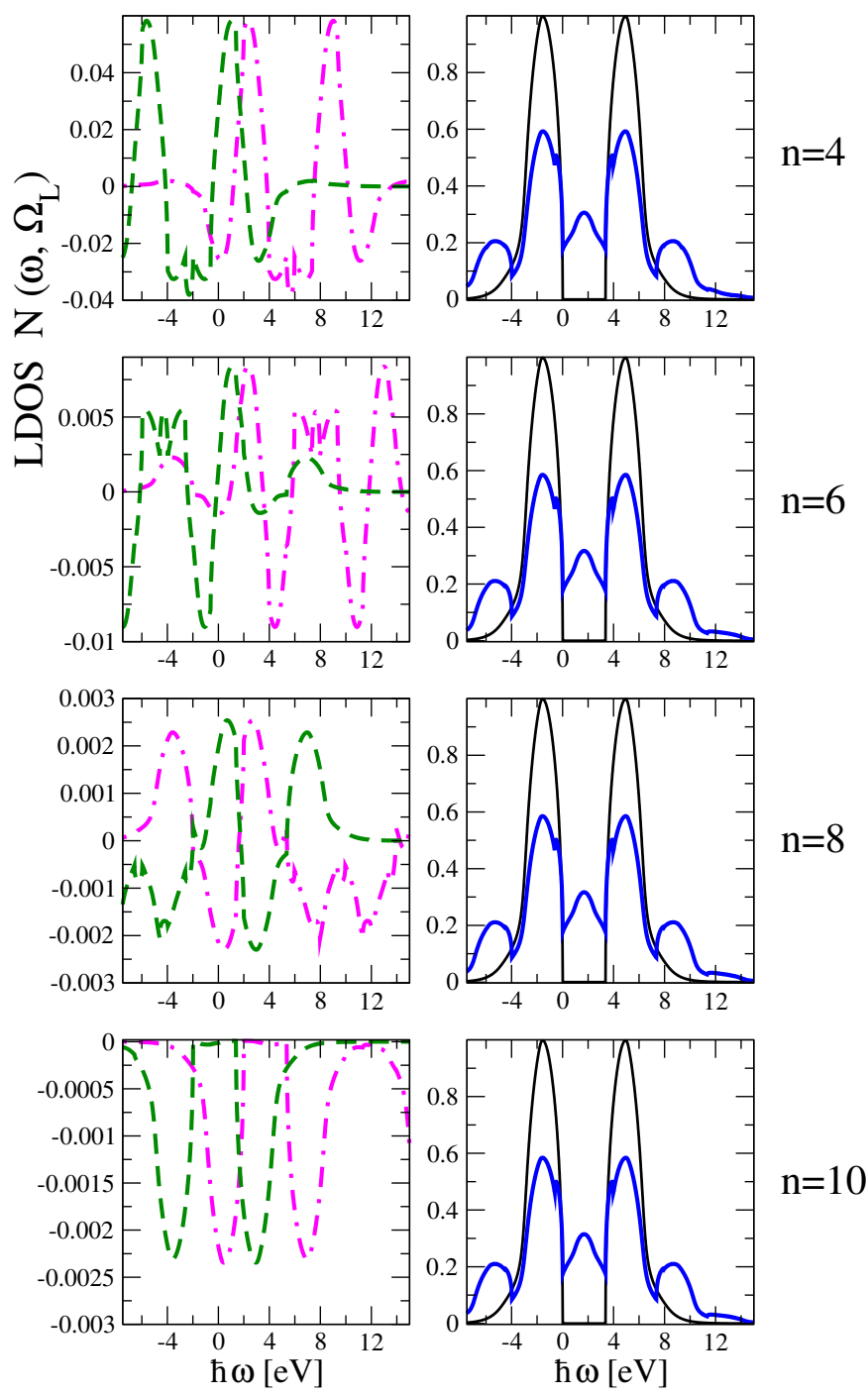

Fig. 7. Floquet contributions and accuracy check of the numerical results for the LDOS of driven semiconductor bulk. The bandgap in equilibrium is $3.38 \mathrm{eV}$, the Fermi edge is $1.69 \mathrm{eV}$. For discussion see section 3.3 . 
states, the LDOS. Consequentially the increase of mathematical and numerical precision of our framework of the Floquet-Keldysh DMFT by increasing the number of Floquet contributions up to the level of the precision of the DMFT has direct consequences for the finding and the accuracy of physical results. Especially the investigation of the coupling of the driven electronic system of the bulk with edge and surface modes will profit from this precision. Since these coupling effects in nanostructure and waveguides are of a high technological importance the advantage of the strictly controllable precision of the Floquet DMFT approach in comparison to time dependent DMFT frameworks is quite obvious.

\section{Conclusions}

We investigated in this article the evolution of Floquet topological quantum states in wide band gap semiconductor bulk as a correlated electronic system in the sense of a generalized Hubbard model with dynamical mean field theory in the non-equilibrium. We found that nonequilibrium modulations induce a non-trivial bandstructure which leads for several frequency ranges to a topologically induced metal phase. This topological bandstructure is a result of the AC-Stark effect. The intersection of Floquet bands and band-edges respectively induces transitions which enhance up- and downconversion effects as well as higher harmonic generation. The absorption coefficient of the semiconductor is topologically modulated and non-trivial transitions at the crossing points of the equilibrium valence band, the Floquet-valence subbands, the Floquet-conduction subbands and the equilibrium conduction band become possible and their significance is depending on the excitation power. We also find the development of novel sub gaps as areas of electromagnetically induced transparency. The number of included Floquet modes is chosen such that energy conservation is numerically guaranteed. This precision is essential in order to achieve predictive results for semiconductor bulk which can be investigated for optoelectronic and magnetooptoelectronic switching as well as for innovative laser systems. Thus the bulk semiconductor under non-equilibrium conditions as such has to be reclassified. It will be of great interest to investigate the interplay of the here derived topological bulk effects with additional surface modifications such as a surface resonance, a polariton coupling, or a surface magneto-optical modulation.

Acknowledgments The authors thank H. Monien, H. Wittel and F. Hasselbach for highly valuable discussions.

\section{Author contributions}

Both authors equally contributed to the presented work. Both authors were equally involved in the preparation of the manuscript. Both authors have read and approved the final manuscript.

\section{References}

1. J. M. Kosterlitz, D. J. Thouless, J. Phys. C : Solid State Phys., 6, 1181 (1973).

2. L. Fu, C. L. Kane, E. J. Mele, Phys. Rev. Lett. 98, 106803 (2007).

3. M. Z. Hasan, C. L. Kane, Rev. Mod. Phys. 82, 3045 (2010).

4. L. Fu, C. L. Kane, Phys. Rev. Lett. 100, 096407 (2008).

5. G. Moore, N. Read, Nucl. Phys. B 360, 362 (1991).

6. N. Goldmann, J. Dalibard, Phys. Rev. X 4, 031027 (2014).

7. I. Zuti, J. Fabian, S. Das Sarma, Rev. Mod. Phys. 76, 323 (2004).

8. C. Nayak, S. H. Simon, A. Stern, M. Freedman, S. Das Sarma, Rev. Mod. Phys. 80, 1083 (2008).

9. M. C. Rechtsman, J. M. Zeuner, Y. Plotnik, Y. Lumer, D. Podolsky, F. Dreisow, S. Nolte, M. Segev, A. Szameit Nature 496, 196 (2013).

10. M. A. Bandres, S. Wittek, G. Harari, M. Parto, J. Ren, M. Segev, D. N. Christodoulides, M. Khajavikhan, Science 359 DOI:10.1126/science.aar4005 (2018).

11. A. Lubatsch, R. Frank, Appl. Sci. 9(12), 2477 (2019).

12. B. A. Bernevig, T. L. Hughes, S.-C. Zhang, Science 314, 1757 (2006).

13. M. König, S. Wiedmann, C. Brüne, A. Roth, H. Buhmann, L. W. Molenkamp, X.-L. Qi, S.-C. Zhang, Science 318, 766 (2007).

14. D. Hsieh, D. Qian, L. Wray, Y. Xia, Y. S. Hor, R. J. Cava, M. Z. Hasan, Nature 452, 970 (2008).

15. Y. Xia, D. Qian, D. Hsieh, L. Wray, A. Pal, H. Lin, A. Bansil, D. Grauer, Y. S. Hor, R. J. Cava, M. Z. Hasan, Nature Physics 5, 398 (2009).

16. H. Zhang, C.-X. Liu, X.-L. Qi, X. Dai, Z. Fang, S.-C. Zhang, Nature Physics 5, 438, (2009).

17. N. H. Lindner, G. Refael, V. Galitski, Nature Physics 7 490-495 (2011).

18. Y. Tenenbaum Katan, D. Podolsky, Phys. Rev. Lett. 110, 016802 (2013).

19. T. Kitagawa, T. Oka, A. Brataas, L. Fu, E. Demler, Phys. Rev. B 84, 235108 (2011).

20. Z. Gu, H. A. Fertig, D. P. Arovas, A. Auerbach, Phys. Rev. Lett. 107, 216601 (2011).

21. Y. H. Wang, H. Steinberg, P. Jarillo-Herrero, N. Gedik Science 342, 453 (2013).

22. M. S. Rudner, N. H. Lindner, E. Berg, M. Levin, Phys. Rev. X 3031005 (2013).

23. N. H. Lindner, D. L. Bergman, G. Refael, V. Galitski Phys Rev. B 87, 235131 (2013).

24. L. Jiang, T. Kitagawa, J. Alicea, A. R. Akhmerov, D. Pekker, G. Refael, J. I. Cirac, E. Demler, M. D. Lukin, P. Zoller, Phys. Rev. Lett. 106, 220402 (2011).

25. L. D'Alessio, M. Rigol, Nat. Comm., 6:8336 (2015).

26. A. G. Grushin, A. Gomez-Leon, T. Neupert, Phys. Rev. Lett. 112, 156801 (2014).

27. E. Bergholtz, Z. Liu, Int. J. Mod. Phys. B 27, 24, 1330017 (2013).

28. G. Floquet, Ann. l' Ecole Norm. Sup. 12, 47 (1883).

29. R. Frank, Phys. Rev. B 85, 195463 (2012).

30. R. Frank, Ann. Phys. (Berlin) 525, No. 1-2, 66-73 (2013).

31. F. H. M. Faisal, J. Z. Kaminski, Phys. Rev. A 56, 1, 748 (1997).

32. M. Grifoni, P. Hänggi, Physics Reports 304, 5, 229 (1998).

33. S. Restrepo, J. Cerrillo, V. M. Bastidas, D. G. Angelakis,

T. Brandes, Phys. Rev. Lett. 117, 250401 (2016). 
34. A. Eckardt, Rev. Mod. Phys. 89, 011004-1 (2017).

35. M. H. Kalthoff, G.S. Uhrig, J. K. Freericks, Phys. Rev. B 98, 035138 (2018).

36. M. A. Sentef, M. Claassen, A. F. Kemper, B. Moritz, T. Oka, J. K. Freericks, T. P. Devereaux, Nat. Comm. 6, 7047 (2015).

37. L. Yuan, S. Fan, Phys. Rev. A 92, 053822 (2015).

38. A. Lubatsch, J. Kroha, Ann. Phys. (Berlin) 18, No. 12, $863-867$ (2009).

39. A. Georges, G. Kotliar, W. Krauth, M. J. Rozenberg, Rev. Mod. Phys. 68 (1), 13 (1996)

40. W. Metzner, D. Vollhardt, Phys. Rev. Lett. 62, 324 (1989)

41. P. Schmidt, H. Monien, arXiv:cond-mat/0202046 (2002).

42. T. Maier, M. Jarrell, T. Pruschke, M. H. Hettler, Rev. Mod. Phys. 77, 1027 (2005).

43. J. K. Freericks, V. M. Turkowski, V. Zlatic, Phys. Rev. Lett. 97, 266408 (2006).

44. N. Tsuji, T. Oka, H. Aoki, Phys. Rev. Lett. 103, 047403 (2009).

45. N. Lin, C. A. Marianetti, A. J. Millis, D. R. Reichman Phys. Rev. Lett. 106, 096402 (2011).

46. D. Zgid, G. K.-L. Chan, The Journal of Chemical Physics, 134 (9), 094115 (2011).

47. H. Aoki, N. Tsuji, M. Eckstein, M. Kollar, T. Oka, P. Werner, Rev. Mod. Phys. 86, 779 (2014).

48. R. Frank, Appl. Phys. B 113, 41 (2013).

49. R. Frank, New J. Phys. 15123030 (2013)

50. M. E. Sorantin, A. Dorda, K. Held, E. Arrigoni, Phys. Rev. B 97, 115113 (2018).

51. W. Hofstetter, T. Qin, J. Phys. B: At. Mol. Opt. Phys. 51 082001 (2018).

52. T. Qin, W. Hofstetter, Phys. Rev. B 97, 125115 (2018).

53. D. A. B. Miller, D. S. Chemla, T. C. Damen, A. C. Gossard, W. Wiegmann, T. H. Wood, C. A. Burrus, Phys. Rev. Lett. 53, 22, 2173-2176 (1984).

54. D. S. Chemla, W. H. Knox, D. A. B. Miller, S. SchmittRink, J. B. Stark, R. Zimmermann, Journal of Luminescence 44, 233-246 (1989).

55. M. Bukov, L. D'Alessio, A. Polkovnikov, Advances in Physics 64, No. 2, 139-226 (2015).

56. J. Zak, Phys. Rev. Lett. 62, 23, 2747-2750 (1989).

57. D. Xiao, M.-C. Chang, Q. Niu, Rev. Mod. Phys. 82, 3, 1959-2007 (2009).

58. D. Gresch, G. Autes, O. V. Yazyev, M. Troyer, D. Vanderbilt, B. A. Bernevig, A. A. Soluyanov, Phys. Rev. B 95 075146 (2017)

59. P.-C. Chang, J. G. Lu, Appl. Phys. Lett. 92, 212113 (2008).

60. P.-C. Chang, C.-J. Chien, D. Stichtenoth, C. Ronning, J. G. Lu, Appl. Phys. Lett. 90, 113101 (2007).

61. F. Huang, Z. Lin, W. Lin, J. Zhang, K. Ding, Y. Wang, Q. Zheng, Z. Zhan, F. Yan, D. Chen, P. Lv, X. Wang, Chinese Science Bulletin 59, 1235 (2014).

62. W. I. Park, Y. H. Jun, S. W. Jung, G.-C. Yi, Appl. Phys. Lett. 82, 6 964-966 (2003).

63. D. Fritsch, H. Schmidt, M. Grundmann, Appl. Phys. Lett. 88, 134104 (2006)

64. H. Dixit, R. Saniz, D. Lamoen, B. Partoens, J. Phys. Condens. Matter 22, 125505 (2010).

65. M. Wegener, Extreme Nonliner Optics, ISBN 3-540-22291X, Springer (2004).

66. R. D. King-Smith and D. Vanderbilt, Phys. Rev. B 47, 1651 (1993).
67. D. Vanderbilt and R. D. King-Smith, Phys. Rev. B 48 4442 (1993).

68. R. Resta, Rev. Mod. Phys., 66, 3, 899-915 (1994).

69. V. Dal Lago, M. Atala, L. E. F. Foa Torres, Phys. Rev. A 92, 023624 (2015).

70. E. Gull, A. Millis, A. I. Lichtenstein, A. N. Rubtsov, M. Troyer, P. Werner, Rev. Mod. Phys., 83, 2, 349 (2011).

71. M. Snoek, I. Titvinidze, C. Toke, K. Byczuk, W. Hofstetter, New J. Phys. 10, 093008 (2008).

72. R. Peters, T. Yoshida, H. Sakakibara, N. Kawakami, Phys. Rev. B 93, 235159 (2016).

73. R. Peters, T. Yoshida, N. Kawakami, Phys. Rev. B 98, 075104 (2018)

74. S. Manmana, A. M. Essin, R. M. Noack, V. Gurarie, Phys. Rev. B 86, 205119 (2012).

75. S. Rachel, Rep. Prog. Phys. 81, 116501 (2018).

76. W. Franz, Z. Naturforschung 13a, 484-489 (1958).

77. L. V. Keldysh, J. Exptl. Theoret. Phys. (USSR) 33 9941003 (1957).

78. L. V. Keldysh, Soviet Physics JETP 6 763-770 (1958).

79. L. V. Keldysh, J. Exptl. Theoret. Phys. (USSR) 471945 1957 (1964).

80. L. V. Keldysh,Soviet Physics JETP 20 1307-1314 (1965). 